\title{
Surface and bulk dissolution properties, and selectivity of DNA-linked nanoparticle assemblies
}

\author{
D. B. Lukatsky and Daan Frenkel \\ FOM Institute for Atomic and Molecular Physics [AMOLF], Kruislaan 407, 1098 SJ Amsterdam, \\ The Netherlands
}

(Received 18 January 2005; accepted 18 March 2005; published online 1 June 2005)

\begin{abstract}
Using a simple mean-field model, we analyze the surface and bulk dissolution properties of DNA-linked nanoparticle assemblies. We find that the dissolution temperature and the sharpness of the dissolution profiles increase with the grafting density of the single-stranded DNA "probes" on the surface of colloids. The surface grafting density is controlled by the linker occupation number, in analogy with quantum particles obeying fractional statistics. The dissolution temperature increases logarithmically with the salt concentration. This is in agreement with the experimental findings [R. Jin, G. Wu, Z. Li, C. A. Mirkin, and G. C. Schatz, J. Am. Chem. Soc. 125, 1643 (2003)]. By exploiting the unique phase behavior of DNA-coated colloids, it should be possible to detect multiple "targets" in a single experiment by essentially mapping the DNA base-pair sequence onto the phase behavior of DNA-linked nanoparticle solution. (C) 2005 American Institute of Physics. [DOI: 10.1063/1.1906210]
\end{abstract}

\section{INTRODUCTION}

DNA-linked and protein-linked nanoparticle solutions ${ }^{1-16}$ represent a novel class of complex liquids. These novel, multicomponent, self-assembling materials are being used for DNA (Refs. 2 and 17) and protein ${ }^{4,6}$ detection. Controlled, DNA-assisted self-assembly is a promising strategy in the bottom-up approach to nonmaterial design. ${ }^{7,8,18,19}$ Both the detection and design applications require the understanding and prediction of the fundamental properties of these complex systems.

In DNA-linked nanoparticle solutions, first introduced experimentally by Mirkin et al. ${ }^{1-3}$ single-stranded DNA (ssDNA) "probe" molecules are grafted onto the surface of gold nanoparticles. There are several species of nanoparticles covered with different, specific sequence ssDNA. These probe ssDNA can specifically bind to the "target," linker ssDNA molecules. The target has a sequence complementary to the probe ssDNA on two (or more) different colloidal species (see Fig. 1). In the experiments of Refs. 1-3, 10, and 11, small gold colloids $(\sim 10-50 \mathrm{~nm})$ and short DNA $(\sim 20-30$ base pairs) were used. The addition of the target ssDNA strands induces a sharp demixing transition ${ }^{20}$ that leads to the aggregation of the nanoparticles coated with the probe ssDNA strands. The aggregates were detected using plasmonresonance optical spectroscopy. ${ }^{3}$

The principal experimental finding ${ }^{2,3,10,11}$ was that these systems consisting of DNA-linked nanoparticles have sharp dissolution profiles (the fraction of dissolved colloids as a function of temperature).$^{20}$ The sharpness of the profiles makes it possible to design the DNA-nanoparticle detection assays with extraordinary selectivity and sensitivity compared to other hybridization-based (e.g., fluorophore-based) assays. $^{2}$ In particular, a single-base mismatch in a 27 base ssDNA sequence was detected with high selectivity in Ref. 2 by monitoring the difference in the DNA-nanoparticles dissolution profiles between the perfectly complementary and mismatched DNA sequences, respectively.

In order to elucidate what controls the properties of DNA-linked nanoparticles, Jin et $a l^{3}$ analyzed the dependence of the dissolution temperature, and the sharpness of the dissolution profiles on the DNA surface coverage, the

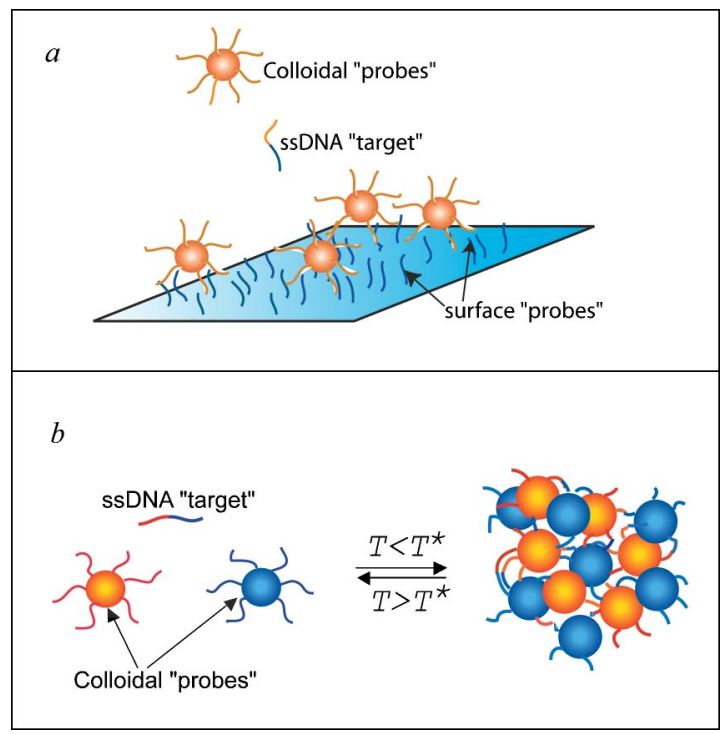

FIG. 1. Schematic representation of DNA-linked colloids in the surface (a) and bulk (b) formats. In (a) the colloids and the surface are covered with ssDNA molecules (these ssDNAs are termed "probes") of specific sequences $A$ and $B$, respectively. In the presence of complementary, linker ssDNA molecules $A^{\prime} B^{\prime}$ (these ssDNAs are termed "targets"), the colloids are adsorbed on the surface. The targets $A^{\prime} B^{\prime}$ have part of the sequence $A^{\prime}$ complementary to the sequence $A$, and another part $B^{\prime}$ complementary to $B$. In (b) we sketch the bulk format with two colloidal species covered with ssDNA of the sequences $A$ and $B$, respectively, and in the presence of target ssDNA linkers $A^{\prime} B^{\prime}$. Below the dissolution temperature $T^{*}$, the colloids aggregate. 
particle size, and the salt concentration. Jin et al. ${ }^{3}$ observed that (i) the higher the ssDNA surface coverage of the colloids, the sharper the dissolution profiles and the higher the dissolution temperature, and (ii) the dissolution temperature increases logarithmically with salt concentration, analogously to the case of a double-stranded DNA (dsDNA) melting temperature. These principal experimental results were obtained both in the case of surface and bulk DNAnanoparticle formats.

We note that there exist similar systems based on phospholipid vesicles (or metal particles) covered with biotin and linked by streptavidin ${ }^{21-28}$ (modeled in Ref. 23); microemulsions linked by polymers with hydrophobic ends ${ }^{29}$ (modeled in Ref. 30); and metal particles linked by metal ions. ${ }^{31-35}$ The principal advantage of the DNA-linked nanoparticles is the ability to control the interactions in a multicomponent colloidal system with an extraordinary selectivity. The selectivity is controlled by the DNA sequences, which makes it possible to tune the strength and the connectivity of the interactions between different species.

In this paper, we use a simple lattice model of reversible gelation in the presence of a solvent to predict the surface and bulk dissolution properties of DNA-linked nanoparticle assemblies. Part of our results were published in the recent paper. $^{36}$ Our results are in qualitative agreement with the experimental findings of Ref. 3 . In the present paper we extend our analysis to the case of multiple DNA targets. The main advantage of the proposed detection method is the ability to detect multiple DNA targets in a single experiment.

The paper is organized as follows: In Sec. II we introduce the model and predict the surface and bulk dissolution properties of DNA-linked nanoparticle assemblies; in Sec. III we study the effect of added salt; in Sec. IV we investigate in detail the effects of competitive linker binding; in Sec. V we propose a novel method for DNA detection with multiple "targets;" and we summarize our results in Sec. VI.

\section{PHASE BEHAVIOR OF DNA-LINKED NANOPARTICLE ASSEMBLIES}

\section{A. Surface hybridization of DNA-linked nanoparticles}

In this section we discuss the process of the targetinduced adsorption of colloids on the surface, Fig. 1(a). Consider a two-dimensional lattice with $N$ lattice sites. The colloids on the surface are in chemical equilibrium with the colloids in the bulk, with $\mu_{A}^{c}$ being the chemical potential of colloids in the bulk. The partition function of the system has the form,

$$
Z=\sum_{N_{A}=0}^{N} \frac{N !}{N_{A} !\left(N-N_{A}\right) !} Q^{N_{A}} e^{\mu_{A}^{c} N_{A} / T}=\left(1+e^{\mu_{A}^{c} / T} Q\right)^{N},
$$

where $N_{A}$ is the number of colloids reversibly absorbed on the surface at a given realization of the grand canonical ensemble, and the combinatorial factor is the number of ways to distribute $N_{A}$ colloids among $N$ lattice sites. In what follows we use the notation $T \equiv k_{B} T$, where $k_{B}$ is the Boltzmann constant. In Eq. (1) we neglected for simplicity the possibility of partial and competitive binding of linkers; we shall discuss this issue below. $Q$ is the linker partition function per colloid on a surface site on the lattice,

$$
Q=\sum_{L=0}^{M} \frac{M !}{L !(M-L) !} e^{L(\mu-\epsilon) / T}=(1+z)^{M},
$$

where $z \equiv \exp [(\mu-\epsilon) / T]$ is the fugacity of linkers, with $\mu$ being the chemical potential of linkers and $\epsilon(T)$ is the temperature-dependent free energy of a single dsDNA molecule connecting a given colloidal particle with the surface site (DNA binding free energy). $M$ is the maximal possible number of dsDNA links that can be formed between a colloid and a surface site. The higher the grafting density of ssDNA molecules on the colloids and on the surface, the higher is the value of $M$. If there is only one pair of probe strands per contact, then this pair can accommodate at most one linker and the bound linkers obey Fermi-Dirac statistics. In general, the maximum number of linkers per contact is a number $M \geqslant 0$. For $\infty>M>1$, the bound linkers obey fractional statistics. ${ }^{37}$ We emphasize that at this level of description, we ignore the interaction between different linkers. We also ignore, for the sake of simplicity, the effect of partial binding of linkers to colloids in solution and to nonbound surface sites. We show below that this effect is insignificant.

In a more general case, a colloid $A$ and a surface site $B$ (or two nearest-neighbor colloids $A$ and $B$ considered in the following section) might have different surface grafting densities (or patches) of probe ssDNA. In this case the numbers $M_{A}$ and $M_{B}$ of probe ssDNA molecules are not equal, and $M$ entering Eq. (2) is the minimum of $M_{A}$ and $M_{B}, M$ $=\min \left(M_{A}, M_{B}\right)$.

In practice, the local coverage of probe molecules may fluctuate. This variation of the ssDNA surface coverage on colloids is the inevitable consequence of the experimental preparation procedure. We assume that the maximum number of linkers per bond obeys Poisson statistics. Moreover, we assume that the values of $M$ for different colloid-surfacesite pairs are uncorrelated. If we average over all possible values of $M$, we obtain the following expression for the grand partition function of linkers (for a given colloid at the surface site),

$$
\bar{Q}=\sum_{M=0}^{\infty} p(M) \sum_{L=0}^{M} \frac{M !}{L !(M-L) !} z^{L}=e^{\bar{M} z},
$$

where $\bar{M}$ is the average value of $M$, and

$$
p(M)=e^{-\bar{M}} \frac{\bar{M}^{M}}{M !}
$$

is the Poisson probability distribution. This probability distribution is applicable even in the case when the number of linkers on colloids varies significantly. ${ }^{38}$ Other types of probability distributions (in particular, the binomial distribution, see the Appendix) can be used for the averaging in Eq. (3), as well. By tuning $\bar{M}$, we change the effective fractional statistics of the linkers. The Fermi-Dirac limit is only recovered when $M$ is fixed, and equal to one.

The average surface fraction of the absorbed colloids, $\sigma$, is found using the partition function, Eq. (1): 


$$
\sigma=\frac{T}{N} \frac{\partial \ln Z}{\partial \mu_{A}^{c}}=\frac{Q e^{\mu_{A}^{c} / T}}{1+Q e^{\mu_{A}^{c} / T}} .
$$

We stress again that the fraction of the reversibly absorbed colloids, $\sigma$, is controlled not only by the dsDNA binding free energy $\epsilon(T)$, but also by the chemical potentials of the colloids $\left(\mu_{A}^{c} / T=\ln [\phi /(1-\phi)]\right.$, where $\phi$ is the bulk fraction of colloids), and the linkers $\left(\mu / T \equiv \mu_{0}=\ln \left[\psi_{b} /\left(1-\psi_{b}\right)\right]\right.$, where $\psi_{b}$ is the bulk fraction of linkers in the reservoir solution). These are simply the chemical potentials of the ideal lattice gas of colloids and linkers, respectively, in the bulk reservoir solution. $^{39,40}$

The configurational entropy of linkers on the surface acts as an additional external field, driving the surface absorption. The higher the fraction of the reversibly absorbed colloids, the higher the configurational entropy of linkers on the surface. This is because the colloids on the surface provide the binding sites for the linkers. The linkers can "hop" between different binding sites, increasing their configurational entropy, and gaining the binding free energy $\epsilon(T)$ per bond. This is illustrated by the semigrand canonical free energy $f$ (canonical with respect to colloids and grand canonical with respect to linkers) obtained from the partition function, Eq. (1),

$$
f=T \sigma \ln \sigma+T(1-\sigma) \ln (1-\sigma)-T \sigma \ln Q,
$$

where the last term represents an effective, linker-induced surface field acting on colloids as an effective chemical potential. We note that the problem of ssDNA linker-induced colloid absorption is similar to the absorption of colloids bearing specific ligands at their surface on the receptorcoated fluid membrane. ${ }^{41}$ The physics of this effect is essentially a generalization of the Langmuir absorption (see also the discussion of surfactant absorption on a liquid-liquid interface, Ref. 42, and ssDNA hybridization on DNA chips, Ref. 43).

The simple analysis presented above, allows one to perform a comparison with the experimental results on the surface dissolution of DNA-linked colloids. ${ }^{2,3}$ The free-energy difference between the double-stranded and melted states of a dsDNA molecule, $\epsilon_{A B}(T) \equiv \epsilon(T)$ constitutes an input to the problem. We adopt the simple form $\epsilon(T)=s\left(T-T_{m}\right)$, where $T_{m}$ is the melting temperature of a dsDNA molecule. ${ }^{44}$ The dissolution curves of DNA-linked colloidal aggregates are shown in Fig. 2. The fraction $\sigma$ of absorbed colloids is plotted as a function of temperature. The maximal possible number of dsDNA links, $M$, between a colloid and a surface site is assumed to be fixed, and we thus used $Q$ given by Eq. (2) to compute Fig. 2. The two curves on each plot, Figs. 2(a) and 2(b), illustrate the effect of a mutation in the target ssDNA: The dsDNA melting temperature $T_{m}$ used to compute the dashed curves is assumed to be $5 \%$ lower than $T_{m}$ for the perfectly matched linker, corresponding to the solid curve. It is remarkable, that the only difference between Figs. 2(a) and 2(b) is the value of $M: M=2$ used in Fig. 2(a) and $M=10$ used in Fig. 2(b). In accordance with the experimental results of Refs. 2 and 3, the dissolution temperature increases with increasing $M$, and so does the sharpness of the dissolution profiles.

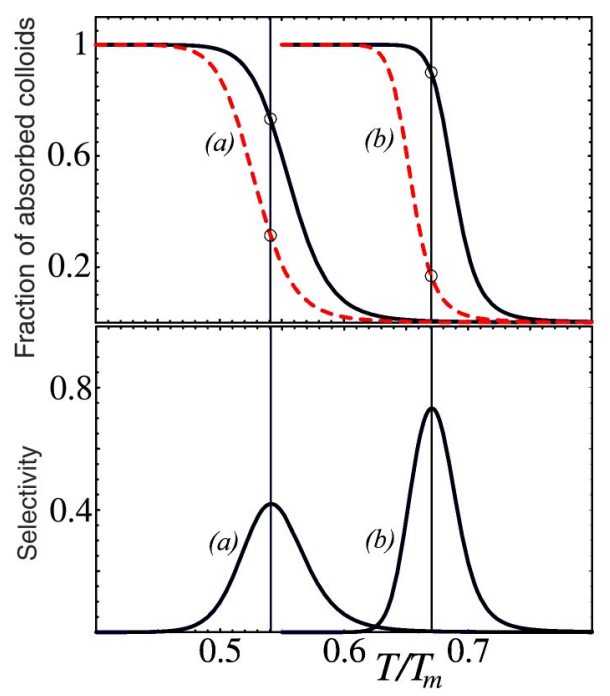

FIG. 2. Top: Surface dissolution profiles of the DNA-linked nanoparticle system. Computed temperature dependence of the fraction $\sigma$ of the absorbed ssDNA-coated colloids. The plots were computed using $\mu_{0}=-4.5, s=10$, $\phi=0.001$. The value of $M$ (assumed to be fixed) is the only difference in computing the plots (a) and (b): $M=2$ was used to compute (a) and $M$ $=10$ was used to compute (b), respectively. The temperature $T$ is relative to the melting temperature $T_{m}$ of the perfectly complementary linker (solid curves). The dashed curves [in each plot (a) and (b)] illustrate the effect of a mismatch in the target DNA. The dsDNA melting temperature for the dashed curve in each plot (corresponding to a mismatch) is 5\% lower than $T_{m}$ for the perfectly matched linker. Bottom: Selectivity plots. The algebraic difference between the solid (perfectly matched linker) and dashed (mismatched linker) curve in each case (a) and (b). The vertical lines and the circles indicate the optimal stringency temperatures. The results presented in this figure agree qualitatively with the experimental measurements of Ref. 2 (see Fig. 2 of Ref. 2).

We stress again that these effects are dominated by both the energetic and entropic contributions of reversibly absorbing colloids and linkers. The higher the surface fraction of absorbed colloids, the higher the configurational entropy of the ssDNA linker molecules on the surface, and thus the higher the entropic cooperativity of the system. The notion of entropic cooperativity means that ssDNA linkers participating in the formation of reversible bonds between colloids and the surface, partially compensate the loss of their translational entropy in the bulk solution by exploring different available binding sites on the surface.

The bottom plots in Fig. 2 show the difference in $\sigma$ between the solid and dashed curves, as a function of temperature. This quantity represents the selectivity of the system. The higher the grafting coverage density of the probe ssDNA molecules on the colloids (represented by $M$ ), the higher the selectivity with respect to a sequence mismatch detection. This result is also in qualitative agreement with the experiments. $^{2,3}$ Qualitatively similar conclusions hold true if the polydispersity in the ssDNA coverage density on colloids is taken into account, Eq. (3). One interesting observation is that the [Poisson-distribution-obeying, Eq. (3)] polydispersity in $M$ leads to slightly sharper dissolution profiles than in the case with fixed $M$, provided that all the other conditions in the solution of colloids and linkers are the same. The sharpness of the profiles in this case is weakly varying with $\bar{M}$. This suggests that not only the ssDNA coverage density 
but also the degree of its polydispersity is a control (stringency) parameter that governs the selectivity of the DNAlinked nanoparticle system, as far as the DNA detection applications are concerned. In particular, this suggests that in order to achieve higher selectivity, the nanoparticles should be manufactured with as high ssDNA coverage density as possible, even if polydispersity of grafting is large.

\section{B. Bulk hybridization of DNA-linked nanoparticles}

In this section we consider a lattice model for a binary mixture of DNA-coated colloids in solution. Each colloidal species is covered with specific ssDNA molecules, in such a way that all possible pairs of colloids can be bound by three types of complementary ssDNA linkers: viz, $A A, B B$, and $A B$.

Colloids of type $A$ and $B$ occupy the nodes of a threedimensional lattice with coordination number $q$. The system is grand canonical with respect to all the components: $\mu_{A}^{c}$ and $\mu_{B}^{c}$ are the chemical potentials in the bulk of colloids of species $A$ and $B$, respectively; and $\mu_{A A}, \mu_{B B}$, and $\mu_{A B}$ are the corresponding chemical potentials of linkers. The grand canonical partition function is

$$
Z=\sum g\left(N_{A}, N_{B}, n_{A B}, n_{A A}, n_{B B}\right) Q_{A B}^{n_{A B}} Q_{A A}^{n_{A A}} Q_{B B}^{n_{B B}} e^{\mu_{A}^{c} N_{A}+\mu_{B}^{c} N_{B} / T},
$$

where $N_{A}$ and $N_{B}$ are the number of colloids $A$ and $B$, respectively, $n_{\alpha \beta}$ is the number of nearest-neighbor $\alpha \beta$ colloidal pairs for a given realization of the grand canonical ensemble, and $g\left(N_{A}, N_{B}, n_{A B}, n_{A A}, n_{B B}\right)$ is the total number of possible configurations with $n_{A B}$ nearest-neighbor $A B$ colloid pairs, $n_{A A}$ nearest-neighbor $A A$ pairs, and $n_{B B}$ nearestneighbor $B B$ pairs, respectively, for given values of $N_{A}$ and $N_{B}$. The sum with respect to $n_{\alpha \beta}$ extends over all possible values consistent with the fact that there are $N_{A}$ and $N_{B}$ colloids $A$ and $B$ present.

The expression for the grand partition function of linkers (for a given $A B$ colloidal pair) is analogous to the one obtained in the surface case, Eq. (2),

$$
Q_{A B}=\left(1+z_{A B}\right)^{M},
$$

where $z_{A B} \equiv \exp \left[\left(\mu_{A B}-\epsilon_{A B}\right) / T\right] . \epsilon_{\alpha \beta}(T)$ is the temperaturedependent binding free energy of a dsDNA molecule connecting a given pair of $\alpha \beta$ colloidal particles. $M$ in Eq. (8) is the maximal possible number of dsDNA bonds that can be formed between a nearest-neighbor $A B$ colloid pair. Similar to the surface case, $M$ represents the surface grafting density of the probe ssDNA molecules on colloids. The linker partition functions $Q_{A A}$ and $Q_{B B}$ are defined analogously to Eq. (8). We emphasize that in our analysis we assume (as we did in the surface case) that the chemical potentials of linkers in solution are fixed: e.g., $\mu_{A B} / T \equiv \mu_{0}=\ln \left[\psi_{A B} /\left(1-\psi_{A B}\right)\right]$, where $\psi_{A B}$ is the fraction of $A^{\prime} B^{\prime}$ ssDNA linker molecules in the reservoir solution. This is a reasonable assumption when there is an excess of linkers in the DNA-colloid solution, which appears to be the case in the experiments of Ref. 3. In particular, the experiments ${ }^{3}$ were performed in the regime with 40 ssDNA linkers (on average) for each $A$ and $B$ colloid pair in solution.
The expression given by Eqs. (7) and (8) can be interpreted as the partition function of a three-state spin model with Hamiltonian,

$$
H=\frac{1}{2} \sum_{\langle i j\rangle} \hat{\sigma}_{i} \hat{J} \hat{\sigma}_{j}-\sum_{i} \hat{\eta} \hat{\sigma}_{i} .
$$

$\hat{\sigma}_{i}=(1,0),(0,1)$, or $(0,0)$ if $A, B$, or a vacancy (solvent) occupies site $i$; and $\hat{\eta}=\left(\mu_{A}^{c}, \mu_{B}^{c}\right)$. The square, symmetric interaction matrix is given by $J_{\alpha \beta}=-T \ln Q_{\alpha \beta}$. We note again that the effective attraction between the colloids induced by linkers is dominated by the energy of DNA binding and the entropy associated with the number of different ways of distributing $L$ linkers over $M$ bonds: in a dense phase, there are simply more bonds. Similar mechanisms are responsible for phase separation in the models of binary hard-core mixtures, ${ }^{45}$ polymer microemulsions, ${ }^{30}$ network-forming microemulsions, ${ }^{46,47}$ and in general, in models of reversible gelation by mobile linkers in a solvent. ${ }^{48}$

The Hamiltonian for a three -component lattice gas model [Eq. (9)] has been studied extensively. ${ }^{49}$ The meanfield expression for the free energy is

$$
\begin{gathered}
f=T \phi_{A} \ln \phi_{A}+T \phi_{B} \ln \phi_{B}+T\left(1-\phi_{A}-\phi_{B}\right) \ln \left(1-\phi_{A}\right. \\
\left.-\phi_{B}\right)+\frac{q J_{A A}}{2} \phi_{A}^{2}+\frac{q J_{B B}}{2} \phi_{B}^{2}+q J_{A B} \phi_{A} \phi_{B},
\end{gathered}
$$

where $\phi_{A}$ and $\phi_{B}$ are the average volume fractions of $A$ and $B$ colloids. The equilibrium phase behavior of the system in the mean-field approximation follows from the analysis of this free energy: ${ }^{49}$ depending on the strength of the interaction between the colloidal species, the system can be in a homogeneous state, or separate into two, or even three, coexisting phases. The model can be generalized to any number of colloidal species and corresponding linkers.

The dissolution curves of the DNA-linked colloidal aggregates are shown in Fig. 3, where we treat the case of only $A^{\prime} B^{\prime}$ linkers added in solution of $A$ and $B$ colloids. The total fraction of colloids in solution, $X$ (e.g., $X_{A}$ is the ratio between the amount of colloids $A$ in dilute phase and the total amount of colloids $A$ in solution), is plotted as a function of temperature for different values of $M$ (computing Fig. 3, we assumed the case without polydispersity in $M$ ). Qualitatively consistent with the measurements of Ref. 3, the dissolution temperature $T^{*}$ of the aggregates [defined by $X\left(T^{*}\right)=1 / 2$ ] increases with increasing surface coverage of ssDNA molecules (represented by the linker occupation number $M$ ) on the colloids, and the sharpness of the profile increases with $M$, as well. The sharpness of the dissolution profiles is the crucial factor in achieving a high selectivity of the DNA mutation analysis. ${ }^{2}$ We thus predict that the selectivity of the system is dominated by the surface grafting density of ssDNA probes on colloids. This is our central result, as far as the applications of this system to the DNA detection are concerned. The critical temperature of the phase separation, $T_{c}$, is determined [from Eq. (10), using standard stability analysis] in the case of symmetric composition of $A$ and $B$ colloids by $q M \ln \left(1+z_{A B}^{c}\right)=8$, where $z_{A B}^{c}$ is the critical value of the linker fugacity. This leads to the following scaling form of the DNA binding free energy at the critical point: 


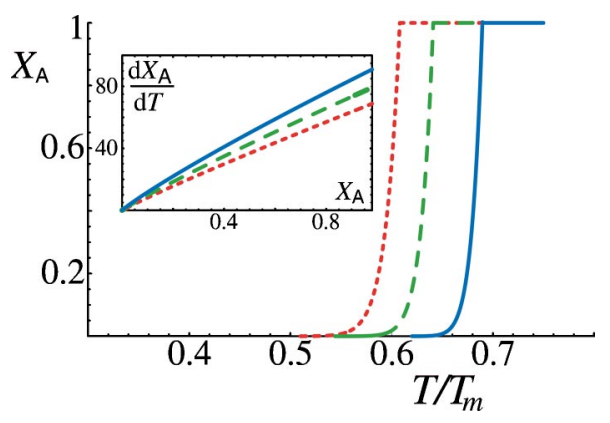

FIG. 3. Bulk dissolution profiles of the binary, symmetric mixture (species $A$ and $B$ of equal average concentrations) of ssDNA-coated colloids and added ssDNA complementary linkers that can form links between $A$ and $B$ colloids, but not between $A$ and $A$, or $B$, and $B$. The total fraction of colloids in the dilute phase, $X_{A}$ is plotted as a function of temperature. Above the demixing temperature, the volume fraction of both the $A$ and $B$ colloids is $0.1 \%$. The three curves illustrate the effect of varying of the ssDNA surface grafting density on colloids ( $M$ is assumed to be fixed in each case, i.e., no polydispersity in $M$ ). The solid curve is obtained with $M=6$, the dashed curve with $M=3$, and the dashed-dotted curve with $M=2$. The chemical potential of ssDNA linkers is $\mu_{0}=-4.5$, and $s=10$. Inset: The slopes of the dissolution profiles are plotted as a function of the total fraction of dissolved colloids. The higher the surface grafting density of ssDNA on colloids (represented by $M$ ), the higher the slope of the dissolution profiles, and the higher the dissolution temperature. This is consistent with the experimental observations of Ref. 3 .

$\epsilon_{A B}\left(T_{c}\right) / T_{c} \sim \ln \psi_{b}+\ln (q M)$. This scaling form is valid in the limit $q M \gg 1$, which is always satisfied in an experiment. Therefore, $T_{c}$ increases with the linker chemical potential, and with the probe DNA coverage density on colloids, represented by $M$.

We note that similar to the surface case described in the preceding section, the Poisson-obeying fluctuations in $M$ result in sharper dissolution profiles as compared with the case of fixed $M$ (provided that all the other parameters are the same). The dissolution temperature $T^{*}$ is slightly higher as compared with the case with fixed $M$.

For the sake of simplicity we neglected the effect of partial ssDNA linker binding to isolated colloids in solution in the above analysis. Moreover, we only considered the dominant effect of the binding between colloids. The effect of partial binding is qualitatively and quantitatively of little significance. We shall address the question of partial and competitive binding in more detail in Sec. IV.

\section{EFFECT OF SALT ON DNA-LINKED NANOPARTICLE ASSEMBLIES}

Let us next consider the effect of the varying of salt concentration on the melting of the DNA-linked colloids. We incorporate the effect of salt only through the salt dependence of the melting temperature of DNA linker molecules. We neglect the electrostatic effects on the strength of the direct colloid-colloid and DNA-colloid interactions. In the experiments of Ref. 3, the salt concentration varied in the range $(0.05-1) \mathrm{M}$. This corresponds to a Debye screening radius $r_{D}$ of about $1.5-0.3 \mathrm{~nm}$. The typical colloid size $(\sim 13-50 \mathrm{~nm})$, and the linker DNA length $(\sim 10 \mathrm{~nm})$ in Ref. 3 are thus about one order of magnitude larger than $r_{D}$. The direct electrostatic interaction between the colloids in solution is thus effectively screened. Even with these approxima-

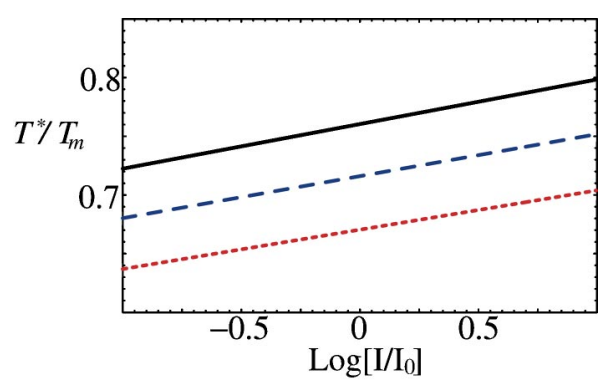

FIG. 4. Effect of added salt on the dissolution temperature $T^{*}$ of the binary mixture of $A$ and $B$ colloids and $A^{\prime} B^{\prime}$ linkers. $T^{*}$ is plotted as a function of $\ln \left(I / I_{0}\right)$, where $I_{0}$ is the salt concentration in the reference state $\left(I_{0}\right.$ $=0.1 \mathrm{M})$. The parameters used to compute this figure are $\mu_{0}=-4.5, s=10$, $q=6, \alpha=0.05, M=5$ (dotted line), $M=10$ (dashed line), and $M=20$ (solid line). Above the dissolution temperature, $\phi_{A}=\phi_{B}=0.001 . T^{*}$ is in the units of the $A^{\prime} B^{\prime}$ linker melting temperature, $T_{m} \equiv T_{m}^{0}$, in the reference state, $I_{0}$ $=0.1 M$.

tions, we can reproduce the dominant effect of added salt on the nano-colloid-ssDNA system. ${ }^{3}$

To predict the effect of the salt concentration on the free energy of the DNA and on its melting temperature, we make use of the relevant theories for polyelectrolytes. ${ }^{50-53}$ Both the theories that focus on Manning condensation ${ }^{50,53}$ and those that use a Poisson-Boltzmann approach, ${ }^{51,52}$ predict that the melting temperature of the DNA melting transition, $T_{m}$, is proportional to the logarithm of the salt concentration, $I$. This result has been verified experimentally (see, e.g., Ref. 54-56). This holds true for both cases of a very short DNA (Ref. 55) (that is, the case relevant for experiments ${ }^{3}$ ), and for very long (tens of thousands of base pairs) DNA. ${ }^{54}$ The mechanism of the effect of salt on the DNA melting transition is mainly entropic: Upon the melting transition, part of the counterions condensed on the highly charged dsDNA molecule get released, reducing the free energy of the system. The higher the salt concentration, the smaller the entropic gain upon the counterion release.

The specific form of the dependence of $\epsilon(T)$ and $T_{m}$ on the salt concentration depends on the fraction of $G C$ and $A T$ base pairs in the DNA sequence. ${ }^{57,54,55}$ For a specific DNA sequence, $T_{m}$ can be practically determined using available experimental data. ${ }^{54-56}$ Here we simply use the correct functional form

$$
T_{m}=T_{m}^{0}[1+\alpha \log c]
$$

where $T_{m}^{0}$ is the DNA melting temperature at a reference state (with a salt concentration $I_{0}=0.1 \mathrm{M}$ ), $\alpha \approx 0.05$, and $c=I / I_{0}$. We assume that the addition of salt affects $\epsilon(T)$ only through $T_{m}$ (this is experimentally verified to be an accurate approximation $^{57}$ ).

The dissolution temperature $T^{*}$ is plotted in Fig. 4, as a function of $\log c$ for different values of $M$. In accordance with the measurements of Ref. 3, $T^{*}$ scales linearly with $\log c$. It is also straightforward to see analytically (using the analysis outlined in Sec. II) that the critical temperature of the phase separation $T_{c} \sim \log c$. We emphasize that this agreement with experimental observations justifies a posteriori the assumption of the insignificance of the direct colloid-colloid electrostatic interactions within the experimentally relevant range of salt concentrations. 
In the surface case, all the same conclusions hold true. There, the dissolution temperature scales linearly with $\log c$ as well.

\section{EFFECT OF COMPETITIVE BINDING OF DNA LINKERS}

\section{A. Competitive binding of linkers to complementary probes}

An interesting situation arises when there is more than one type of ssDNA linkers that compete for binding to the (partially) complementary probes. This applies both in the case of surface and bulk binding. Specifically, consider the linker partition function in the bulk case (the linkers bind to two colloids $A$ and $B$ ) discussed in Sec. II B. The difference with respect to the previously considered cases is that now there are two types of linkers $A^{\prime} B^{\prime}$ and $A^{\prime \prime} B^{\prime \prime}$. Both of these linkers bind to the complementary probes, but with different affinity. For example, we assume that the $A^{\prime} B^{\prime}$ linker is perfectly complementary and $A^{\prime \prime} B^{\prime \prime}$ linker is mismatched with respect to the probe ssDNA grafted on $A$ and $B$ colloids. In this case of two competing linker types, the linker partition function $Q$ per colloid pair is

$$
Q=\sum_{L=0}^{M} z_{1}^{L} \sum_{L_{1}=0}^{M-L} z_{2}^{L_{1}} \frac{M !}{L ! L_{1} !\left(M-L-L_{1}\right) !},
$$

where

$$
\begin{aligned}
& z_{1}=\exp \left(\frac{\mu_{A^{\prime} B^{\prime}}-\epsilon_{A^{\prime} B^{\prime}}}{T}\right), \\
& z_{2}=\exp \left(\frac{\mu_{A^{\prime \prime} B^{\prime \prime}}-\epsilon_{A^{\prime \prime} B^{\prime \prime}}}{T}\right),
\end{aligned}
$$

with $\mu_{A^{\prime} B^{\prime}}$ and $\mu_{A^{\prime \prime} B^{\prime \prime}}$ being the chemical potentials, and $\epsilon_{A^{\prime} B^{\prime}}$ and $\epsilon_{A^{\prime \prime} B^{\prime \prime}}$ being the binding free energies of linkers $A^{\prime} B^{\prime}$ and $A^{\prime \prime} B^{\prime \prime}$, respectively.

The result of the summation in Eq. (12) is

$$
Q=\left(1+z_{1}+z_{2}\right)^{M} \text {. }
$$

In the case of the Fermi-Dirac linker statistics, $M=1$, the partition function of linkers takes the simple form: $Q=1$ $+z_{1}+z_{2}$. We can take easily into account the polydispersity in the grafting density of ssDNA grafted on colloids. The averaging of Eq. (14) with respect to the Poisson distribution, $p(M)=\exp (-\bar{M}) \bar{M}^{M} / M$ ! gives the result: $\bar{Q}=\exp \left[\bar{M}\left(z_{1}+z_{2}\right)\right]$.

The overall effect of the competitive binding is thus the renormalization of the effective, linker-induced surface field in the surface case, Eq. (1), or the effective intercolloid potential in the bulk case:

$$
J_{A B}=-T \ln Q \text {. }
$$

This analysis suggests a possible method to enhance the sensitivity of DNA mutation detection systems. Suppose one has only a limited amount of the target (this is especially important in the case when the target is a protein and not a DNA due to the difficulty in replicating a protein). One can add first a known amount of a specific (known sequence) ssDNA target. At a specific temperature, just below the dis- solution temperature of the system without unknown target, one thus needs very little amount of the target to induce demixing of the colloids. The dissolution curves can then be analyzed and compared with the dissolution curves obtained with the target ssDNA of a known sequence. The sequence of the unknown target can thus be derived.

\section{B. Partial binding of DNA linkers}

Thus far, we ignored the possibility of partial binding of ssDNA linkers to dissolved colloids. In the case of $A$ and $B$ colloids, and $A^{\prime} B^{\prime}$ linkers, the linkers can partially bind to dissolved colloids, without establishing a bond between them. This turns out to be a minor effect, as we shall show below.

In the bulk case, the partition function is

$$
\begin{aligned}
Z= & \sum g\left(N_{A}, N_{B}, n\right) Q_{A B}^{n} \Psi_{A}^{K\left(N_{A}-n\right)} \Psi_{B}^{K\left(N_{B}-n\right)} \\
& \times e^{\left(N_{A} \mu_{A}^{c}+N_{B} \mu_{B}^{c}\right) / T},
\end{aligned}
$$

where $\Psi_{A} \equiv 1+z_{A}$ with $z_{A} \equiv \exp \left[\left(\mu-\epsilon_{A}\right) / T\right]$, and with $\mu$ $\equiv \mu_{A B}$; and $\Psi_{B}$ is defined analogously to $\Psi_{A} \cdot \epsilon_{A}(T)$ is the DNA binding energy of a linker bound to a colloid $A$ (but not bound to $B$ ), and $K$ is the number of ssDNA probes on a colloid (in experiments, ${ }^{3}$ about 100 ssDNA probe strands were grafted on a single $13 \mathrm{~nm}$ Au particle-one strand per $5 \mathrm{~nm}^{2}$ ). The linker partition function $Q_{A B}$ per nearestneighbor $A B$ colloid pair takes now into account the partial binding of linkers to the probes not occupied by the linkers fully bound to the colloids,

$$
\begin{aligned}
Q_{A B}= & \sum_{L=0}^{M} \frac{M !}{L !(M-L) !} z_{A B}^{L} \sum_{L_{1}=0}^{K-L} \sum_{L_{2}=0}^{K-L} \frac{(K-L) !}{L_{1} !\left(K-L-L_{1}\right) !} \\
& \times z_{A}^{L_{1}} \frac{(K-L) !}{L_{2} !\left(K-L-L_{2}\right) !} z_{B}^{L_{2}} .
\end{aligned}
$$

The first sum in Eq. (17) counts the total number of ways to distribute linkers among $M$ possible bonds between a nearest-neighbor pair of colloids $A$ and $B$. The second and third sums count the total number of ways to distribute linkers among the available partial bonds on colloids $A$ and $B$, respectively. The result is

$$
\begin{aligned}
Q_{A B}= & \left(1+z_{A}\right)^{K-M}\left(1+z_{B}\right)^{K-M} \times\left(1+z_{A B}+z_{A}+z_{B}\right. \\
& \left.+z_{A} z_{B}\right)^{M} .
\end{aligned}
$$

We assumed here that the coverage density (represented by $K)$ is the same for both colloidal species. The $\Psi_{A}^{K\left(N_{A}-n\right)}$, $\Psi_{B}^{K\left(N_{B}-n\right)}$ terms in Eq. (16) count all possible ways to distribute linkers among $K\left(N_{A}-n\right)$ and $K\left(N_{B}-n\right)$ possible, partial probes on the isolated (i.e., not bound) $A$ and $B$ colloids, respectively, in the grand canonical ensemble.

The mean-field free energy resulting from the partition function, Eq. (16), is

$$
\begin{aligned}
f= & T \phi_{A} \ln \phi_{A}+T \phi_{B} \ln \phi_{B}+T\left(1-\phi_{A}-\phi_{B}\right) \ln \left(1-\phi_{A}\right. \\
& \left.-\phi_{B}\right)+q J_{A B} \phi_{A} \phi_{B}-T \phi_{A} K \ln \Psi_{A}-T \phi_{B} K \ln \Psi_{B},
\end{aligned}
$$

where 


$$
J_{A B}=-T M \ln \left[\frac{1+z_{A B}+z_{A}+z_{B}+z_{A} z_{B}}{\Psi_{A} \Psi_{B}}\right]
$$

Therefore, taking into account the effect of partial ssDNA target binding leads to the renormalization of the effective, linker-induced, colloid-colloid interaction potential, and to a shift of the chemical potentials of the colloids. Practically, we predict that the effect of the partial binding is insignificant, as far as the phase behavior of DNA-nanoparticle solutions is concerned. This is because within the experimentally relevant range of the parameters, $z_{A B} \gg z_{A, B}$ and $z_{A B} \gg z_{A} z_{B}$. In particular, assuming that $\epsilon_{A}(T)=\epsilon_{B}(T) \simeq \epsilon_{A B}(T) / 2, s=10, \mu_{0}$ $=-4.5, \phi_{A}=\phi_{B}=0.001, M=10$ (assuming fixed $M$ ), and $K$ $=100$, one obtains [by analyzing the free energy, Eq. (19)], that the dissolution temperature, $T^{*}$, is only different (lower) by about $1 \%$ if the effect of partial binding is taken into account. This example provides an a posteriori confirmation that the effect of partial linker binding to isolated colloids in solution is negligible. Moreover, we stress that the predicted small effect due to partial linker binding might be in practice sensitive to the DNA excluded volume effects, and these effects should be taken into account in order to compute accurate corrections to the results presented in this section.

\section{Nanoparticle self-assembly with long and flexible DNA linkers}

Thus far, we assumed that the linkers were effectively rigid objects that could connect only one pair of probes. It is interesting to consider how flexibility of the linker affects the phase behavior of the nanoparticles. In practice, this situation could be experimentally realized by a long (double-stranded) spacer in the single-stranded linker. ${ }^{9}$

We now consider a lattice model where we take into account two principal effects of flexibility: (i) enhanced entropic cooperativity of flexible linkers and (ii) the possibility of binding to the next-nearest neighbors.

(i) Due to its flexibility, the linker DNA can bind between different bonds within the nearest-neighbor colloidcolloid pair. In particular, the total number of ways $L$ linkers (i.e., $A^{\prime} B^{\prime}$ linkers) can bind between $M_{1}$ probe $A$ ssDNA on one colloid and $M_{2}$ probe $B$ ssDNA on the nearest-neighbor colloid is now

$$
\frac{M_{1} !}{L !\left(M_{1}-L\right) !} \frac{M_{2} !}{\left(M_{2}-L\right) !} \text {. }
$$

In the limit $L \ll M_{1}, L \ll M_{2}$, this expression can be simplified as follows:

$$
\frac{M_{1} !}{L !\left(M_{1}-L\right) !} \frac{M_{2} !}{\left(M_{2}-L\right) !} \simeq \frac{M_{1}^{L} M_{2}^{L}}{L !} .
$$

The partition function of linkers can now be computed in a simple, closed form,

$$
Q=\sum_{L=0}^{M_{1}} \frac{M_{1}^{L} M_{2}^{L}}{L !} z^{L}=\frac{\exp \left(M_{1} M_{2} z\right) \Gamma\left(1+M_{1}, M_{1} M_{2} z\right)}{M_{1} !}
$$

where $z$ is the fugacity of DNA linkers, $\Gamma$ is the incomplete gamma function, and we assumed that $M_{1}<M_{2}$. The fugacity $z=\exp \left\{\left[\mu-\epsilon(T)-3 R^{2} / 2 R_{G}^{2}\right] / T\right\}$, where $R$ is the nearestneighbor lattice spacing, and $R_{G}$ is the linker DNA radius of gyration. ${ }^{39,58}$ We assumed that the linker DNA represents an ideal polymer coil with the free energy $F_{L}=F_{0}+3 R^{2} / 2 R_{G}^{2}$ (see Refs. 39 and 58), where the reference free energy $F_{0}$ is incorporated in the definition of the chemical potential $\mu$. The assumption of the ideal polymer coil is a reasonable approximation if the contour length of DNA linkers significantly exceeds the dsDNA persistence length of $50 \mathrm{~nm}$ (this is the case, e.g., $\lambda$ DNA with a contour length of $16 \mu \mathrm{m})$. We also assumed, as we did above, that linker DNA molecules do not interact mutually.

In the limit of high surface grafting density $M_{1} \gg 1$. Using the asymptotic form of the gamma function at $M_{1} M_{2} z$ $\gg 1$,

$$
\Gamma\left(1+M_{1}, M_{1} M_{2} z\right) \simeq e^{-M_{1} M_{2} z}\left(M_{1} M_{2} z\right)^{M_{1}},
$$

we obtain

$$
Q \simeq e^{M_{1}}\left(M_{2} z\right)^{M_{1}},
$$

and finally the effective, nearest-neighbor colloid-colloid interaction potential:

$$
J_{A B} \simeq-T M_{1}\left[1+\ln \left(M_{2} z\right)\right] .
$$

This formula constitutes our main result in this section. The linker flexibility thus leads to enhanced entropic attraction between the colloids. The experimental realization of the system that provides the strongest entropic attraction could thus involve colloids densely grafted with short ssDNA probes. The linker ssDNA targets in this case are composed of a long spacer with the recognition strands (complementary to the probes) at the ends. We stress that, as follows from Eq. (26), in order to have the strong entropic attraction, both colloidal species should have a high grafting density, since the interaction potential $J_{A B}$ is proportional to the number of bonds, $M_{1}$, of the patch with the lowest surface grafting density, $M_{1}<M_{2}$, and only logarithmically scales with $M_{2}$.

(ii) The DNA linkers can bind not only between the nearest-neighbor complementary colloids, but also between the next-nearest neighbors. The partition function for the system of $A$ and $B$ colloids and $A^{\prime} B^{\prime}$ long and flexible linkers can be approximately written in the form:

$$
Z=\sum g\left(N_{A}, N_{B}, n, m\right) Q^{n} \Omega^{m} e^{\left(N_{A} \mu_{A}^{c}+N_{B} \mu_{B}^{c}\right) / T},
$$

where $n$ and $m$ are the numbers of $A B$ nearest-neighbors and next-nearest-neighbors colloidal pairs, respectively, for a given configuration of $N_{A}$ and $N_{B}$ colloids. The sum in Eq. (27) extends over all possible values of $N_{A}, N_{B}, n$, and $m$ for all possible realizations of the grand-canonical ensemble. The linker grand-canonical partition function, $\Omega$, per nextnearest-neighbor $A B$ colloidal pair is given by Eq. (23), provided that $z$ is substituted with $z_{\text {next }}=\exp \{[\mu-\epsilon(T)$ 
$\left.-3 \alpha^{2} R^{2} / 2 R_{G}^{2}\right] / T$, where $\alpha R$ is next-nearest-neighbor lattice spacing, and we assume that $M_{1}$, and $M_{2}$ are the number of bonds for the next-nearest-neighbor colloidal pair (as we did for the nearest-neighbor pair).

The Hamiltonian of the spin model, corresponding to the partition function, Eq. (27), is

$$
H=\frac{1}{2} \sum_{(n n)} \hat{\sigma}_{i} \hat{J} \hat{\sigma}_{j}+\frac{1}{2} \sum_{(n n n)} \hat{\sigma}_{i} \hat{I} \hat{\sigma}_{j}-\sum_{i} \hat{\sigma}_{i} \hat{\eta}
$$

where the spin variables are defined analogously to Eq. (9). The first and the second sums in Eq. (28) are taken with respect to the nearest-neighbor $(n n)$ and the next-nearestneighbor ( $n n n)$ sites, respectively. In the present case where only $A^{\prime} B^{\prime}$ linkers are present, the symmetric, quadratic matrices $J_{\alpha \beta}$ and $I_{\alpha \beta}$ have only two nonzero (antidiagonal) elements, where

$$
I_{A B}=-T \ln \Omega \simeq-T M_{1}\left[1+\ln \left(M_{2} z_{\text {next }}\right)\right] .
$$

The principal conclusion is that the presence of long DNA linkers leads to an enhanced effective attraction between the colloids. This is easy to understand intuitively: long and flexible DNA linkers simply increase the range of the colloidcolloid interactions, and also increase the configurational entropy of the system, as compared with the shorter linkers. The mean-field free energy of the system with long DNA linkers has the form similar to Eq. (10),

$$
\begin{aligned}
f= & T \phi_{A} \ln \phi_{A}+T \phi_{B} \ln \phi_{B}+T\left(1-\phi_{A}-\phi_{B}\right) \ln \left(1-\phi_{A}\right. \\
& \left.-\phi_{B}\right)+\left(q J_{A B}+\tau I_{A B}\right) \phi_{A} \phi_{B},
\end{aligned}
$$

where $q$ and $\tau$ are the numbers of nearest neighbors and next-nearest neighbors on the lattice, respectively. It is straightforward to take into account more colloidal species with more possible combinations of DNA linkers, simply by increasing the number of components in Eq. (30).

\section{PHASE SEPARATION AND DNA SEQUENCE ANALYSIS}

As far as DNA (Ref. 2) (or protein ${ }^{4}$ ) detection is concerned, the principal experimental challenge is to distinguish a specific target molecule (or molecules) in the solution containing many different species of similar molecules. The presence of competing species obscures the measurements, and thus constitutes the major experimental obstacle to achieve a high selectivity and sensitivity of detection.

In Ref. 36 we proposed a possible scenario to distinguish a specific target ssDNA $A^{\prime} B^{\prime}$ by monitoring the composition of phases in a three-component system of $A, B$, and $C$ colloids. We noticed very generally that if the target $A^{\prime} B^{\prime}$ binds to $A B\left(A^{\prime} B^{\prime} / A B\right)$ and $A C\left(A^{\prime} B^{\prime} / A C\right)$ with slightly different affinities (i.e., $A B$ and $A C$ have similar sequences which are only different by a mismatch, either a single base or multiple base), one can distinguish the sequence of the target by monitoring the composition difference of $B$ and $C$ colloids in a dense (or dilute) phase. The colloids can be, e.g., fluorescently labeled, and their compositions can thus be determined.

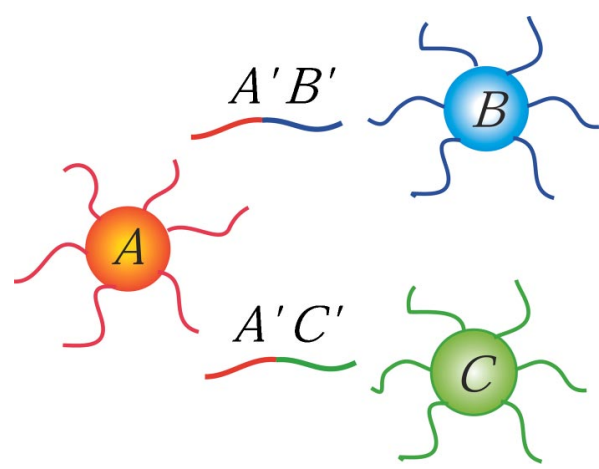

FIG. 5. Schematic representation of the three-component system of $A, B$, and $C$ colloidal species covered with ssDNA of three different probe sequences, respectively, and two species of ssDNA targets $A^{\prime} B^{\prime}$ and $A^{\prime} C^{\prime}$. $A^{\prime} B^{\prime}$ complementary binds $A B$, and also binds $A C$ with slightly weaker affinity. Similarly, $A^{\prime} C^{\prime}$ complementary binds $A C$, and also binds $A B$ with slightly weaker affinity.

Even a small difference in the $A^{\prime} B^{\prime}$ binding free energy with respect to $A B$ and $A C$ can induce a large difference in the composition of phases.

Here we generalize the approach of Ref. 36 to the case of multiple targets. We show that one can detect different targets in a single experiment. In particular, we consider a three-component solution of colloids. $A, B$, and $C$, and two linker species $A^{\prime} B^{\prime}$ and $A^{\prime} C^{\prime}$ (see Fig. 5). We assume that linkers $A^{\prime} B^{\prime}$ are perfectly complimentary to $A B$, and $A^{\prime} C^{\prime}$ are perfectly complementary to $A C$. We stress that $A^{\prime} B^{\prime}$ binds $A C$, and $A^{\prime} C^{\prime}$ binds $A B$, i.e., there is a competitive binding of linkers.

The partition function of the solution of linkers and colloids has the following form:

$$
\begin{aligned}
Z= & \sum g\left(N_{A}, N_{B}, N_{C}, n_{A B}, n_{A C}\right) Q_{A B}^{n_{A B}} Q_{A C}^{n_{A C}} \\
& \times e^{\mu_{A}^{c} N_{A}+\mu_{B}^{c} N_{B}+\mu_{C}^{c} N_{C} / T},
\end{aligned}
$$

where the linker partition functions $Q_{A B}$ and $Q_{A C}$ take into account competitive linker binding (see Sec. IV A):

$$
Q_{A B}=\left(1+z_{A B}+y_{A C}\right)^{M}
$$

and

$$
Q_{A C}=\left(1+z_{A C}+y_{A B}\right)^{M},
$$

with the following definitions of fugacities of direct binding $\left(z_{A B}, z_{A C}\right)$, and competitive binding $\left(y_{A B}, y_{A C}\right)$ :

$$
\begin{aligned}
& z_{A B}=\exp \left(\frac{\mu_{A B}-\epsilon_{A B}}{T}\right), \\
& z_{A C}=\exp \left(\frac{\mu_{A C}-\epsilon_{A C}}{T}\right), \\
& y_{A B}=\exp \left(\frac{\mu_{A B}-\epsilon_{A B}^{A C}}{T}\right), \\
& y_{A C}=\exp \left(\frac{\mu_{A C}-\epsilon_{A C}^{A B}}{T}\right) .
\end{aligned}
$$




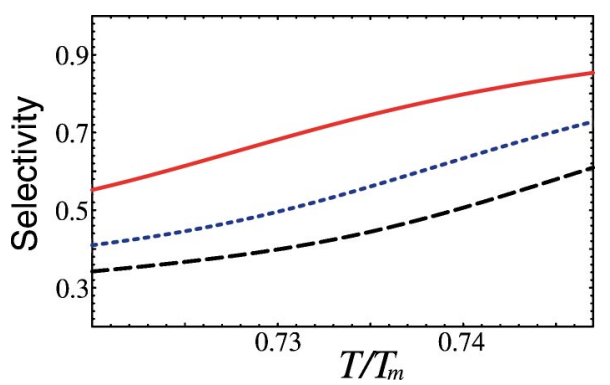

FIG. 6. Selectivity plot. Selectivity of the phase behavior of a ternary suspension consisting of $A, B$, and $C$ colloidal species and two types of ssDNA linkers $A^{\prime} B^{\prime}$ and $A^{\prime} C^{\prime}$ (see text). The binding free energies of linkers are assumed to have the form $\epsilon_{A B}=s\left(T-T_{m}\right), \epsilon_{A C}=s\left(T-T_{m}^{A C}\right), \epsilon_{A B}^{A C}=\epsilon_{A C}^{A B}=s(T$ $-T_{m}^{\text {comp }}$ ), where $T_{m}$ and $T_{m}^{A C}$ are the melting temperatures of $A^{\prime} B^{\prime}$ and $A^{\prime} C^{\prime}$ linkers, respectively, and $T_{m}^{\text {comp }}$ is the melting temperature of the competitive binding. The curves show that the presence of the linkers induces a large relative concentration differences $\left(\phi_{B}-\phi_{C}\right) /\left(\phi_{B}+\phi_{C}\right)$ of $B$ and $C$ colloidal species in the dense phase. Above the demixing temperature, the concentration of all colloidal species is the same, $\phi_{A}=\phi_{B}=\phi_{C}=0.001$. The parameters used in to compute this figure are $\mu_{A B} / T=\mu_{A C} / T \equiv \mu_{0}=-4.5, s=10, q=6$, $M=10$, and $T_{m}^{A C}=0.98 T_{m}$. The three curves illustrate the effect of the strength of the competitive binding: $T_{m}^{\text {comp }}=0.9 T_{m}$ (solid line), $T_{m}^{\text {comp }}$ $=0.94 T_{m}($ dotted line $)$, and $T_{m}^{\text {comp }}=0.96 T_{m}$ (dashed line). $T$ is expressed in the units of $T_{m}$ of the linker $A^{\prime} B^{\prime}$ that binds $A B$.

Here $\mu_{A B}$ and $\mu_{A C}$ are the chemical potentials of linkers $A^{\prime} B^{\prime}$ and $A^{\prime} C^{\prime}$, respectively; $\epsilon_{A B}$ and $\epsilon_{A C}$ are the corresponding DNA binding free energies $\left(A^{\prime} B^{\prime} / A B\right.$ and $A^{\prime} C^{\prime} / A C ; \epsilon_{A B}^{A C}$ is the binding free energy of the linker of the type $A^{\prime} B^{\prime}$ that binds to $A C\left(A^{\prime} B^{\prime} / A C\right)$; and $\epsilon_{A C}^{A B}$ is the DNA binding free energy of the linker $A^{\prime} C^{\prime}$ that binds to $A B\left(A^{\prime} C^{\prime} / A B\right)$. The latter two binding free energies take into account the competitive binding of linkers $A^{\prime} B^{\prime}$ and $A^{\prime} C^{\prime}$. We assume that

$$
\epsilon_{A B}^{A C} \approx \epsilon_{A C}^{A B} \gtrsim \epsilon_{A C} \gtrsim \epsilon_{A B} .
$$

This corresponds to the scenario where the two types of linkers have similar sequences, and are only different by a (few) base-pair mismatches. We also assume here that linker $A^{\prime} B^{\prime}$ binds slightly stronger to $A B$, compared with $A^{\prime} C^{\prime}$ to $A C$.

The mean-field free energy is

$$
\begin{aligned}
f= & T \phi_{A} \ln \phi_{A}+T \phi_{B} \ln \phi_{B}+T \phi_{C} \ln \phi_{C}+T\left(1-\phi_{A}-\phi_{B}\right. \\
& \left.-\phi_{C}\right) \ln \left(1-\phi_{A}-\phi_{B}-\phi_{C}\right)+q J_{A B} \phi_{A} \phi_{B}+q J_{A C} \phi_{A} \phi_{C},
\end{aligned}
$$

where $J_{A B}=-T \ln Q_{A B}$ and $J_{A C}=-T \ln Q_{A C}$.

The addition of the targets $A^{\prime} B^{\prime}$ and $A^{\prime} C^{\prime}$ induces a two-phase separation in the system. Our principal observation is that the composition of the dense phase (and, by implication, the composition of the remaining solution) depends on the nature of the target ssDNAs. In particular, even in the case of similar-sequence targets [the DNA binding free energies of the targets $\epsilon_{A B}$ and $\epsilon_{A C}$ are similar in magnitude, Eq. (35)], we generically observe a large concentration difference between $B$ and $C$ colloids in the phase-separated system.

The characteristic mean-field phase diagram for this case is shown in Fig. 6 in terms of the concentration differences (selectivity plot) between $B$ and $C$ colloids. The initial concentrations of $A, B$, and $C$ colloids are chosen to be equal. Our main prediction is that the multiple targets can be de- tected simply by monitoring the concentration differences of the species $B$ and $C$ in either a dense or dilute phase. In the case shown in Fig. 6 the melting temperature $T_{m}$ of $A^{\prime} B^{\prime} / A B$ DNA is assumed to be only $2 \%$ higher than $T_{m}^{A C}$ of $A^{\prime} C^{\prime} / A C$. The principal observation is that even in the case of strong competitive binding $\left(T_{m}^{\text {comp }} \simeq 0.96 T_{m}\right.$, see the caption of Fig. 6 ), the selectivity of the system is as large as $50 \%$. The higher the strength of the competitive binding, the lower the selectivity of the system, Fig. 6.

The method can be generalized to discriminate between a larger number of possible ssDNA targets, simply by increasing the number of colloidal probe species. In essence, the method allows us to map the microscopic base-pair sequence of multiple targets ssDNA onto the macroscopic phase behavior of the DNA-linked nanoparticle solution. We emphasize finally that the proposed method is not limited to only ssDNA targets. It can be applied also to detect protein targets. In this case the probe molecules grafted on colloids are protein receptors - the molecules that specifically bind a protein target of interest. For example, this system has been recently realized experimentally ${ }^{4}$ using magnetic microparticle probes grafted with antibodies that bind specifically an antigen.

\section{SUMMARY AND CONCLUSIONS}

In summary, we investigated the dissolution properties of DNA-linked nanoparticle assemblies using a simple model of colloidal phase separation induced by ssDNA linkers. We predict that the experimentally observed dissolution properties of these assemblies in the surface ${ }^{2,3}$ and bulk bu $^{3,10}$ formats can be explained by surface absorption and phase separation, respectively. In particular, in the bulk, the observed aggregated phase ${ }^{3}$ corresponds to a dense phase of a phase-separated system of colloids and ssDNA linkers. In agreement with experimental findings, ${ }^{3}$ we predict that the dissolution temperature and the sharpness of the dissolution profiles increase with the surface grafting density of ssDNA "probes" on the surface of colloids. Within the framework of our model, the grafting density is controlled by the linker occupation number $M$. As far as applications of this system to DNA detection analysis are concerned, we predict that the surface grafting density of the probes is the dominant factor that governs the selectivity of the system. We predict that the dissolution temperature of the assemblies increases logarithmically with the salt concentration. This is also in agreement with experimental findings. ${ }^{3}$

Most importantly for the applications, we propose a novel method for DNA detection analysis. The method allows one to map the microscopic DNA sequence onto the macroscopic phase behavior of a DNA-nanoparticle system. We noticed very generally, that in the multicomponent system of colloidal probes and multiple linkers that bind different pairs of the probe species, a small difference in the binding affinity of linkers translates into a large difference in the phase composition of the phase-separated colloidal probes. By labeling the colloids (e.g., fluorescently), one can distinguish between different ssDNA "targets" in a single experi- 
ment by simply monitoring the composition of colloids in different phases.

\section{ACKNOWLEDGMENTS}

The authors are grateful to B. Bozorgui, E. Eiser, W. Poon, and T. Schmatko for useful discussions and collaborative work, and to $\mathrm{H}$. Tepper for critical reading of the manuscript and for valuable comments. They thank C.-H. Kiang for sending them the manuscript prior to publication. D.L. also thanks S. Safran for useful discussions. The work of the FOM Institute is part of the research program of FOM and is made possible by financial support from The Netherlands Organization for Scientific Research (NWO).

\section{APPENDIX}

In this appendix we give the expression for the partition function $\bar{Q}$ of the linkers per colloid-surface site (or colloidcolloid nearest-neighbor pair) in the case when the number of DNA bonds, $M$, obeys the binomial probability distribution,

$$
P(M \mid \bar{M})=\frac{\bar{M} !}{M !(\bar{M}-M) !} p^{M}(1-p)^{\bar{M}-M},
$$

where $\bar{M}$ is the maximum possible value of $M$, and $p \leqslant 1$ is the probability of success in a given trial. The averaging of $Q$, Eq. (2) with $P(M \mid \bar{M})$ results:

$$
\bar{Q}=(1+p z)^{\bar{M}}
$$

Of course, in the limit $p=1, \bar{Q}$ is equal to $Q$, given by Eq. (2) with $M=\bar{M}$.

The sharpness of the dissolution profiles is practically independent of $\bar{M}$ for the binomially distributed fluctuations of $M$, and the dissolution temperature, obviously, decreases with decreasing $p$.

${ }^{1}$ C. A. Mirkin, R. L. Letsinger, R. C. Mucic, and J. J. Storhoff, Nature (London) 382, 607 (1996).

${ }^{2}$ T. A. Taton, C. A. Mirkin, and R. L. Letsinger, Science 289, 1757 (2000). ${ }^{3}$ R. Jin, G. Wu, Z. Li, C. A. Mirkin, and G. C. Schatz, J. Am. Chem. Soc. 125, 1643 (2003).

${ }^{4}$ J.-M. Nam, C. S. Thaxton, and C. A. Mirkin, Science 301, 1884 (2003).

${ }^{5}$ S.-J. Park, A. A. Lazarides, J. J. Storhoff, L. Pesce, and C. A. Mirkin, J. Phys. Chem. B 108, 12375 (2004).

${ }^{6}$ K. B. Lee, E. Y. Kim, C. A. Mirkin, and S. M. Wolinsky, Nano Lett. 4, 1869 (2004).

${ }^{7}$ R. C. Mucic, J. J. Storhoff, C. A. Mirkin, and R. L. Letsinger, J. Am. Chem. Soc. 120, 12674 (1998).

${ }^{8}$ C. M. Soto, A. Srinivasan, and B. R. Ratna, J. Am. Chem. Soc. 124, 8508 (2002).

${ }^{9}$ V. T. Milam, A. L. Hiddessen, J. C. Crocker, D. J. Graves, and D. A. Hammer, Langmuir 19, 10317 (2003).

${ }^{10}$ C.-H. Kiang, Physica A 321, 164 (2003).

${ }^{11}$ Y. Sun, N. C. Harris, and C.-H. Kiang, Physica A (in press).

${ }^{12}$ N. C. Harris and C.-H. Kiang (unpublished).

${ }^{13}$ P. L. Biancaniello, A. J. Kim, and J. C. Crocker, Phys. Rev. Lett. 94, 058302 (2005)
${ }^{14}$ M.-P. Valignat, O. Theodoly, J. C. Crocker, W. B. Russel, and P. M. Chaikin, Proc. Natl. Acad. Sci. U.S.A. 102, 4225 (2005).

${ }^{15}$ A. V. Tkachenko, Phys. Rev. Lett. 89, 148303 (2002).

${ }^{16}$ G. P. Goodrich, M. R. Helfrich, J. J. Overberg, and C. D. Keating, Langmuir 20, 10246 (2004).

${ }^{17}$ T. G. Drummond, M. G. Hill, and J. K. Barton, Nat. Biotechnol. 21, 1192 (2003).

${ }^{18}$ N. C. Seeman, Nature (London) 421, 427 (2003).

${ }^{19}$ E. Katz and I. Willner, Angew. Chem., Int. Ed. 43, 6042 (2004).

${ }^{20}$ In Refs. 2 and 3, the word "melting" is used to describe the process of dissolution of the DNA-linked colloidal aggregates. In the present work, we avoid the use of terms "melting" and "freezing" for this transition and use instead "dissolution" and "demixing," as we argue that it is, in fact, more closely related to a liquid-liquid phase separation. This phase transition should also be distinguished from the melting of ssDNA linker molecules bound to complementary ssDNA grafted to colloids.

${ }^{21}$ S. Chiruvolu, S. Walker, J. Israelachvili, F.-J. Schmitt, D. Leckband, and J. A. Zasadzinski, Science 264, 1753 (1994).

${ }^{22}$ E. T. Kisak, M. T. Kennedy, D. Trommeshauser, and J. A. Zasadzinski, Langmuir 16, 2825 (2000).

${ }^{23}$ I. Farbman-Yogev, Y. Bohbot-Raviv, and A. Ben-Shaul, J. Phys. Chem. A 102, 9586 (1998)

${ }^{24}$ S. Cobbe, S. Connolly, D. Ryan, L. Nagle, R. Eritja, and D. Fitzmaurice, J. Phys. Chem. B 107, 470 (2003).

${ }^{25}$ S. Connolly, S. Cobbe, and D. Fitzmaurice, J. Phys. Chem. B 105, 2222 (2001).

${ }^{26}$ P. Vermette, S. Taylor, D. Dunstan, and L. Meagher, Langmuir 18, 505 (2002).

${ }^{27}$ W. Shenton, S. A. Davis, and S. Mann, Adv. Mater. (Weinheim, Ger.) 11, 449 (1999)

${ }^{28}$ N. T. Kim Thanh and Z. Rosenzweig, Anal. Chem. 74, 1624 (2002).

${ }^{29}$ M. Filali, M. J. Ouazzani, E. Michel, R. Aznar, G. Porte, and J. Appell, J. Phys. Chem. B 105, 10528 (2001).

${ }^{30}$ A. Zilman, J. Kieffer, F. Molino, G. Porte, and S. A. Safran, Phys. Rev. Lett. 91, 015901 (2003).

${ }^{31}$ A. N. Shipway, M. Lahav, R. Gabai, and I. Willner, Langmuir 16, 8789 (2000).

${ }^{32}$ I. Willner and B. Willner, Pure Appl. Chem. 74, 1773 (2002).

${ }^{33}$ S.-Y. Lin, S.-W. Liu, C.-M. Lin, and C.-H. Chen, Anal. Chem. 74, 330 (2002).

${ }^{34}$ E. C. Constable, W. Meier, C. Nardin, and S. Mundwiler, Chem. Commun. (Cambridge) 16, 1483 (1999).

${ }^{35}$ Y. Kim, R. C. Johnson, and J. T. Hupp, Nano Lett. 1, 165 (2001).

${ }^{36}$ D. B. Lukatsky and D. Frenkel, Phys. Rev. Lett. 92, 068302 (2004).

${ }^{37}$ In our previous work (Ref. 36), we considered a special case of linker statistics when the DNA "probe" molecules are indistinguishable.

${ }^{38}$ L. D. Landau and E. M. Lifshitz, Statistical Physics, 3rd ed. (Pergamon, London, 1980).

${ }^{39}$ P.-G. de Gennes, Scaling Concepts in Polymer Physics (Cornell University Press, Ithaca, 1988).

${ }^{40}$ S. A. Safran, Statistical Thermodynamics of Surfaces, Interfaces, and Membranes (Addison-Wesley, Reading, MA, 1994).

${ }^{41}$ D. van Effenterre and D. Roux, Europhys. Lett. 64, 543 (2003)

${ }^{42}$ H. Diamand and D. Andelman, Europhys. Lett. 34, 575 (1996).

${ }^{43}$ A. Halperin, A. Buhot, and E. B. Zhulina, Biophys. J. 86, 718 (2004)

${ }^{44}$ The expression for the free energy difference that we employ $[\epsilon(T)=s(T$ $-T_{m}$ ), where $\left.s \sim N|\Delta S|\right]$, represents the first term in the expansion of the exact free energy, $\epsilon(T) \sim N(\Delta H-T \Delta S)$, where $N$ is the number of base pairs in a DNA molecule, $\Delta H$ and $\Delta S$ are, respectively, the enthalpy and entropy difference between the helix and coil states at a given temperature per base pair (see, e.g., chapter 41 of Refs. 58 and 57).

${ }^{45}$ D. Frenkel and A. A. Louis, Phys. Rev. Lett. 68, 3363 (1992).

${ }^{46}$ T. Drye and M. E. Cates, J. Chem. Phys. 96, 1367 (1992).

${ }^{47}$ T. Tlusty, S. A. Safran, and R. Strey, Phys. Rev. Lett. 84, 1244 (2000).

${ }^{48}$ A. Coniglio, H. E. Stanley, and W. Klein, Phys. Rev. Lett. 42, 518 (1979); Phys. Rev. B 25, 6805 (1982).

${ }^{49}$ S. Krinsky and D. Mukamel, Phys. Rev. B 11, 399 (1975); D. Furman, S. Dattagupta, and R. B. Griffiths, ibid. 15, 441 (1977); T. Schilling and G. Gompper, J. Chem. Phys. 117, 7284 (2002).

${ }^{50}$ G. C. Manning, Biopolymers 15, 1333 (1975). 
${ }^{51}$ M. D. Frank-Kamenetskii, V. V. Anshelevich, and A. V. Lukashin, Sov. Phys. Usp. 151, 595 (1987).

${ }^{52}$ J. Bond, C. Anderson, and M. T. Record, Biophys. J. 67, 825 (1994).

${ }^{53}$ C. R. Cantor and P. R. Schimmel, Biophysical Chemistry, Part III (Freeman, New York, 2002), Chap. 22.

${ }^{54}$ R. D. Blake and S. G. Delcourt, Nucleic Acids Res. 26, 3323 (1998).
${ }^{55}$ R. Owczarzy, P. M. Vallone, F. J. Gallo, T. M. Paner, M. J. Lane, and A. S. Benight, Biopolymers 44, 217 (1997).

${ }^{56}$ J. SantaLucia, Jr., Proc. Natl. Acad. Sci. U.S.A. 95, 1460 (1998).

${ }^{57}$ I. Rouzina and V. A. Bloomfield, Biophys. J. 77, 3242 (1999).

${ }^{58}$ A. Yu. Grosberg and A. R. Khokhlov, Statistical Physics of Macromolecules (AIP, New York, 1994). 